\title{
Lateral versus central tendon-splitting approach to insertional Achilles tendinopathy: a retrospective study
}

\author{
Zhan $\underline{X i a}^{1}$, MBBS, Khye Soon Andy $\underline{\text { Yew }}{ }^{1}$, BEng, PhD, Ting Karen $\underline{\text { Zhang }}{ }^{2}$, BSc, Inderjeet Singh $\underline{\text { Rikhraj }}{ }^{1}$, FRCS, FAmS
}

\begin{abstract}
INTRODUCTION This study aimed to compare operative outcomes between the lateral approach (LA) and the central approach (CA) to insertional Achilles tendinopathy (IAT).

METHODS We retrospectively reviewed patients who underwent surgical treatment for IAT using the LA or CA. Patients' demographic data, postoperative complications and satisfaction rate were reviewed. Clinical outcomes were prospectively assessed preoperatively, at three months postoperatively and at the last visit, including patients' visual analogue scale (VAS) scores for pain, American Orthopaedic Foot and Ankle Society (AOFAS) Ankle-Hindfoot Scale scores and 36-item Short Form Health Survey (SF-36) scores.

RESULTS There were 39 patients in the LA group and 32 in the CA group. In each group, average VAS and AOFAS Scale scores improved significantly. SF-36 scores improved in most parameters. No significant difference in baseline score; average AOFAS Scale score at each visit; and mean VAS score preoperatively and at last visit; satisfaction rate; and overall complication rate were observed between the groups. The mean VAS score in the LA group at postoperative three months was significantly lower than that in the CA group $(2.7 \pm 2.5$ vs. $4.4 \pm 3.0 ; p=0.016)$. There were significantly more cases of delayed wound healing in the LA group than in the CA group ( $2.6 \%$ vs. $15.6 \% ; p=0.049$ ).

CONCLUSION Both approaches had comparable outcomes for IAT in terms of functional improvement, pain relief, overall enhancement of patients' health condition and overall postoperative complication rate. The LA provided better short-term pain relief and reduced delayed wound healing compared with the CA.
\end{abstract}

Keywords: Achilles tendon, central tendon-splitting approach, lateral approach, operative procedure, tendinopathy

\section{INTRODUCTION}

Insertional Achilles tendinopathy (IAT), a disease at the insertional area of the Achilles tendon into the calcaneum, is characterised by pain, swelling and impaired performance. ${ }^{(1)}$ It is a common cause of posterior heel pain and is more prevalent in young and active populations, particularly athletes. ${ }^{(2)}$ The annual incidence of this disorder in top-level runners is $7 \%-9 \%{ }^{(3)}$ Although the exact pathogenesis of IAT remains unknown, increased mechanical strain, advancing age and decreased vascularity are often deemed as causative factors. ${ }^{(4-6)}$ Clinical presentations include localised pain over the posterior heel, swelling, burning and stiffness, which usually are aggravated by exertion. ${ }^{(7)}$ Radiology may show calcifications at the insertion of the Achilles tendon, with oedema and thickening of the insertional area of the tendon that may be seen on magnetic resonance imaging. ${ }^{(8,9)}$

Nonoperative treatments, such as activity and footwear modification, eccentric training and extracorporeal shockwave therapy, are the primary management. ${ }^{(10-12)}$ Should nonoperative treatments fail, operative treatments may be considered. Surgery typically includes partial or total detachment of the Achilles tendon, excision of retrocalcaneal bursa, resection of prominent superior calcaneal tuberosity, debridement of the diseased tendon and reattachment of the tendon. ${ }^{(6,12)} \mathrm{A}$ tendon transfer may be considered. ${ }^{(4,13)}$ The surgery can be done either by open or endoscopic means. Open surgery may be performed with a single longitudinal incision (placed either laterally or medially) or a central tendon-splitting incision. A single transverse incision, a J-shaped incision, and a combination of lateral and medial incisions have also been described. However, to the best of our knowledge, the most effective open operative approach has yet to be defined.

The objective of this study was to retrospectively compare the outcomes of operative treatment of IAT between the lateral approach (LA) and the central tendon-splitting approach (CA), using prospectively collected data.

\section{METHODS}

This study was approved by our institutional review board and informed consent was obtained. A retrospective review was done of patients who underwent operative treatment for IAT between August 2007 and May 2015 at Singapore General Hospital, Singapore. All patients were diagnosed with IAT based on preoperative clinical, imaging and intraoperative findings of the affected heel. The operative procedure included partial detachment of the Achilles tendon, debridement of the diseased tendon, excision of retrocalcaneal bursa, resection of prominent superior calcaneal tuberosity and reattachment of the tendon. Patients were excluded if they had a previous Achilles tendon rupture or other previous surgeries, had infection over the same heel, or had undergone operative correction with complete detachment of the Achilles tendon.

Patients were categorised into the LA group and CA group based on the two different open operative approaches. All patients' 
medical records were retrieved. Data extracted included gender, age, body mass index (BMI), length of conservative management, duration of surgery, length of hospitalisation and postoperative complications. Preoperative data that was prospectively collected included: visual analogue scale (VAS) scores to measure preoperative and postoperative subjective pain during activity (with 0 indicating no pain and 10 indicating extremely severe pain); American Orthopaedic Foot and Ankle Society (AOFAS) Ankle-Hindfoot Scale scores; and 36-item Short Form Health Survey (SF-36) scores to evaluate functional outcome. In the SF-36, eight parameters are scored on a scale of 0 to 100 , with 100 being the maximal level of possible function. Scores of a subjective question involving postoperative satisfaction were also prospectively recorded. Patients were asked to rate the overall result of the surgery ( $1=$ excellent, 2 = very good, $3=$ good, 4 = fair, 5 = poor and $6=$ terrible).

All operations were performed by the senior author (i.e. Rikhraj IS). Patients were given prophylactic antibiotics at the induction of anaesthesia and for 24 hours postoperatively, together with adequate analgesics. A thigh tourniquet was applied for all patients. For the lateral approach, as described by Lin et $\mathrm{al}^{\left({ }^{(14)}\right.}$ the patient was placed in the lateral position under anaesthesia. A lateral incision parallel to the lateral border of the Achilles tendon was marked out (Fig. 1) and made. The Achilles tendon was exposed and partially detached from the lateral aspect, and the diseased part of the tendon was debrided. For the central tendon-splitting approach, as described by McGarvey et $\mathrm{al}^{(15)}$ and $\mathrm{Xia}$ et $\mathrm{al},{ }^{(16)}$ the patient was placed in the prone position with a midline incision marked out (Fig. 2). The Achilles tendon was centrally split and partially detached from the calcaneum along the tendon insertion from the midline to the lateral half.

For both groups, the debridement of fibrous degeneration and calcification dictated the amount of tendon that remained. This was decided by the surgeon through repetitive palpation of the tendon. The retrocalcaneal bursa was excised to expose the superior aspect of the calcaneum, and Haglund's prominence was resected using an oscillating saw and rongeurs. Subsequently, the Achilles tendon was reattached to the posterior calcaneum using two suture anchors (HEALIX BR ${ }^{T M}$ Anchor with Orthocord, Corkscrew FT II [Arthrex, Naples, FL, USA] or Insite ${ }^{\text {TM }}$ FT Suture Anchor System [Tornier, Bloomington, MN, USA]), with the foot in plantigrade position. The wound was thoroughly irrigated, followed by closure of subcutaneous tissue and the skin.

Sutures over the operative wound were removed between postoperative day (POD) 14 and POD 21 based on the condition of wound healing. All patients followed the same rehabilitation protocol. A non-weight-bearing plaster cast was applied with the foot in plantigrade position for 1-3 weeks after the operation, followed by a weight-bearing-as-tolerated controlled ankle motion walker boot until six weeks postoperatively. Footwear was subsequently allowed. Following removal of the cast or the walker boot, rehabilitation was initiated, consisting of Achilles tendon stretching, ankle range of motion exercises and proprioception training. The rehabilitation was continued until functional goals were met. We prospectively recorded postoperative VAS, AOFAS

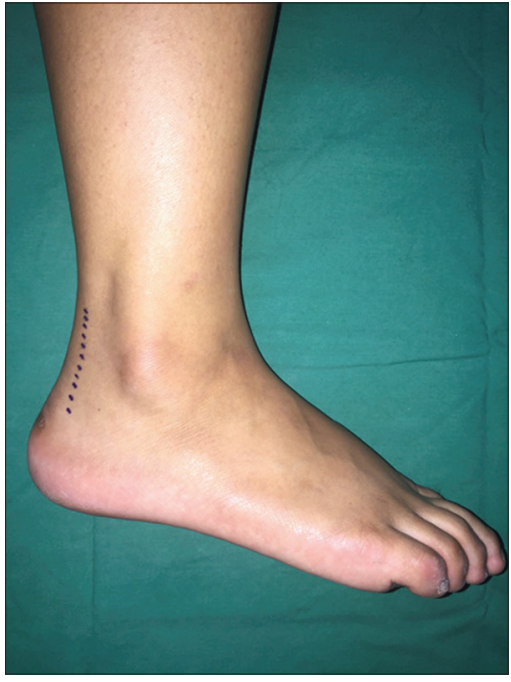

Fig. 1 Photograph shows the lateral approach, parallel to the anterior border of the Achilles tendon, marked out on the heel of one of the subjects.

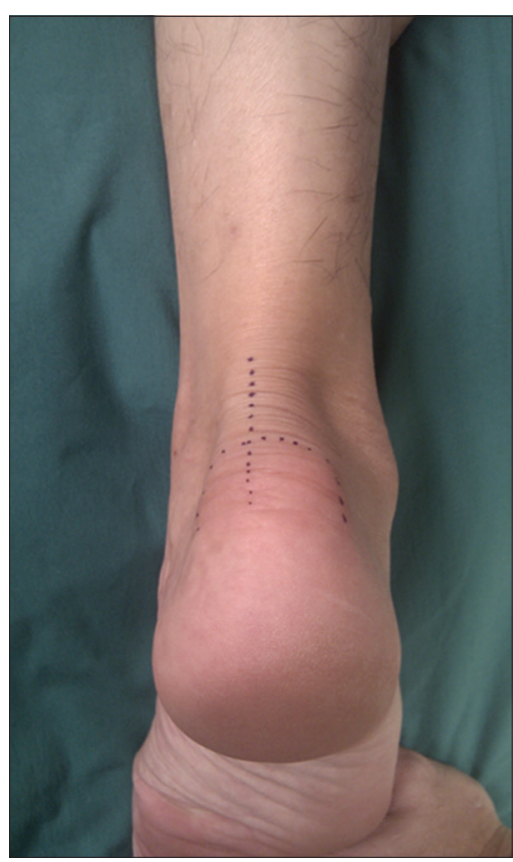

Fig. 2 Photograph shows the central tendon-splitting approach marked out on the heel of one of the subjects.

Ankle-Hindfoot scale and SF-36 scores at the three-month postoperative visit and the last visit, as well as satisfaction rate at the last visit.

Statistical analysis was performed using IBM SPSS Statistics version 20.0 (IBM Corp, Armonk, NY, USA). Two-tailed paired $t$-test and one-way analysis of variation were used to assess the differences in preoperative and postoperative data. MannWhitney $U$ test and chi-square test were applied to evaluate the differences between the two groups. The level of significance was set at $\mathrm{p}<0.05$.

\section{RESULTS}

The data of 84 patients who were diagnosed with insertional Achilles tendinopathy (i.e. 84 heels) and had undergone operative treatment were reviewed. 13 patients who had previous surgeries 
Table I. Demographic data of the patients.

\begin{tabular}{|c|c|c|c|}
\hline \multirow[t]{2}{*}{ Parameter } & \multicolumn{2}{|c|}{ No. (\%) } & \multirow[t]{2}{*}{ p-value } \\
\hline & LA group $(n=39)$ & CA group $(n=32)$ & \\
\hline Age at surgery* $(y r)$ & $55 \pm 12(20.2-75.7)$ & $58 \pm 11(25.9-78.9)$ & 0.229 \\
\hline Gender & & & 0.271 \\
\hline Female & $28(72)$ & $19(59)$ & \\
\hline Male & $11(28)$ & $13(41)$ & \\
\hline Body mass index ${ }^{\dagger}$ & $28.1 \pm 3.7$ & $28.9 \pm 3.9$ & 0.367 \\
\hline \multicolumn{4}{|l|}{ Side of operated heel } \\
\hline Right & $23(59)$ & $19(59)$ & 0.973 \\
\hline Left & $16(41)$ & $13(41)$ & \\
\hline Duration of surgery $^{+}(\mathrm{min})$ & $44.3 \pm 8.6$ & $50.9 \pm 20.3$ & 0.446 \\
\hline Length of hospital stay ${ }^{\dagger}$ (day) & $1.6 \pm 1.3$ & $1.7 \pm 1.2$ & 0.108 \\
\hline Follow-up duration* (mth) & $16.1 \pm 7.6(10-45)$ & $16.2 \pm 4.9(12-27)$ & 0.283 \\
\hline
\end{tabular}

Data is presented as *mean \pm standard deviation (range), tmean \pm standard deviation. CA: central approach; LA: lateral approach

on the same heel were excluded. The remaining 71 patients were allocated into the LA group (39 heels) and CA group (32 heels). Patient demographics are summarised in Table I. There was no significant difference between the two groups in terms of age, gender, preoperative BMI, side of operated heel, operating duration and length of hospitalisation. The average follow-up duration was $16.1 \pm 7.6$ (range 10-45) months and $16.2 \pm 4.9$ (range 12-27) months in the LA group and CA group, respectively.

In the LA group, mean VAS score improved significantly from $7.1 \pm 1.7$ preoperatively to $2.7 \pm 2.5$ at the three-month postoperative visit $(p<0.001)$ and $1.4 \pm 2.1$ at the last visit $(p<0.001)$. Similarly, in the CA group, the preoperative mean VAS score of $7.4 \pm 1.7$ also significantly improved to $4.4 \pm 3.0$ at three months postoperatively $(p<0.001)$ and to $0.7 \pm 1.6$ at the last visit $(p<0.001)$. When comparing VAS scores between the LA and CA groups, a significant difference was observed at the three-month postoperative visit $(p=0.016)$, while no significant difference was seen preoperatively $(p=0.432)$ and at the last visit $(p=0.142)$.

Average AOFAS Ankle-Hindfoot Scale scores in the LA group increased significantly from $43.1 \pm 15.3$ preoperatively to 68.5 \pm 19.8 at the three-month postoperative visit $(p<0.001)$ and $88.7 \pm 14.6$ at the last visit $(p<0.001)$. In the CA group, scores increased significantly from $43.0 \pm 17.6$ preoperatively to $60.8 \pm$ 21.2 at the three-month postoperative visit $(p<0.001)$ and $91.8 \pm$ 9.8 at the last visit $(p<0.001)$. When comparing average AOFAS Ankle-Hindfoot Scale scores between the LA and CA groups, no significant difference was seen at the preoperative $(p=0.945)$, three-month postoperative ( $p=0.094)$ or last visit $(p=0.554)$. SF-36 scores improved in most of the parameters, but there was no significant difference between the two groups postoperatively. These assessments are summarised in Table II.

Postoperative complications for both study groups are summarised in Table III. Complications in the LA group included painful scarring $(7.7 \%)$, wound sensation impairment $(7.7 \%)$, delayed wound healing $(2.6 \%)$, superficial wound infection $(2.6 \%)$ and overgrown osteophytes at the heel requiring a secondary operation $(2.6 \%)$, while those in the CA group included painful scarring $(6.3 \%)$, wound sensation impairment $(3.1 \%)$ and delayed wound healing (15.6\%). There was no superficial wound infection in the CA group. No Achilles tendon rupture or deep wound infection was noted in both groups. Delayed wound healing, which occurs when a wound takes a longer-thanexpected time to completely heal, was defined in this study as complete healing of a heel wound that takes longer than three weeks. We observed that the occurrence of delayed wound healing in the CA group was significantly higher as compared with the LA group $(p=0.049)$. For all other complications, no significant difference was detected. The overall complication rate was comparable, at $23.1 \%$ in the LA group and $25.0 \%$ in the CA group $(p=0.850)$. The overall satisfaction rate was $87.2 \%$ in the LA group and $87.5 \%$ in the CA group $(p=0.968)$.

\section{DISCUSSION}

Although different open approaches have been described for operative management of IAT, there is a paucity of literature on the comparison between different approaches, and the optimal approach has not been defined. The current study reviewed 71 heels that were diagnosed with IAT and treated surgically, comparing the outcomes of 39 heels that were operated on using the LA with that of 32 heels for which the CA was used. To the best of our knowledge, this is the study with the largest sample size that focuses on comparing the effectiveness and complications of the LA and CA for IAT.

The LA to the posterior heel was popularised by Palmer ${ }^{(17)}$ and is a common approach for IAT. It provides direct exposure of the posterolateral heel, particularly superolateral calcaneal exostosis. The major disadvantage of this approach is sural nerve injury, leading to numbness or paraesthesia of the skin. ${ }^{(18)}$ Yodlowski et $\mathrm{al}^{(19)}$ reported that as many as $34.1 \%$ of the patients had altered sensation of the skin. However, the incidence of impaired wound sensation in their study was $7.7 \%$, with no statistically significant difference in wound sensation impairment between the two approaches. Our relatively low rate of nerve injury may be explained by the fact that soft tissue was meticulously handled to minimise nerve injury. 
Table II. Clinical outcomes of the patients.

\begin{tabular}{|c|c|c|c|}
\hline \multirow[t]{2}{*}{ Parameter } & \multicolumn{2}{|c|}{ Mean \pm SD } & \multirow[t]{2}{*}{ p-value } \\
\hline & LA group & CA group & \\
\hline \multicolumn{4}{|l|}{ VAS score } \\
\hline Preoperative & $7.1 \pm 1.7$ & $7.4 \pm 1.7$ & 0.432 \\
\hline 3-mth postoperative & $2.7 \pm 2.5$ & $4.4 \pm 3.0$ & $0.016^{*}$ \\
\hline Last visit & $1.4 \pm 2.1$ & $0.7 \pm 1.6$ & 0.142 \\
\hline p-value & $<0.001^{*}$ & $<0.001^{*}$ & \\
\hline \multicolumn{4}{|c|}{ AOFAS Ankle-Hindfoot Scale score } \\
\hline Preoperative & $43.1 \pm 15.3$ & $43.0 \pm 17.6$ & 0.945 \\
\hline 3-mth postoperative & $68.5 \pm 19.8$ & $60.8 \pm 21.2$ & 0.094 \\
\hline Last visit & $88.7 \pm 14.6$ & $91.8 \pm 9.8$ & 0.554 \\
\hline p-value & $<0.001^{*}$ & $<0.001^{*}$ & \\
\hline \multicolumn{4}{|l|}{ SF-36 score } \\
\hline \multicolumn{4}{|l|}{ Physical function } \\
\hline Preoperative & $58.7 \pm 23.0$ & $53.0 \pm 27.6$ & 0.375 \\
\hline Last visit & $78.0 \pm 18.1$ & $78.0 \pm 15.0$ & 0.678 \\
\hline$p$-value & $<0.001^{*}$ & $0.008^{*}$ & \\
\hline \multicolumn{4}{|c|}{ Role limitations (physical) } \\
\hline Preoperative & $24.4 \pm 36.0$ & $24.2 \pm 38.4$ & 0.840 \\
\hline Last visit & $72.4 \pm 39.7$ & $76.6 \pm 39.1$ & 0.577 \\
\hline$p$-value & 0.235 & 0.059 & \\
\hline \multicolumn{4}{|l|}{ Bodily pain } \\
\hline Preoperative & $34.2 \pm 16.3$ & $34.3 \pm 21.4$ & 0.753 \\
\hline Last visit & $65.7 \pm 26.6$ & $67.1 \pm 22.5$ & 0.806 \\
\hline$p$-value & 0.152 & $0.033^{*}$ & \\
\hline \multicolumn{4}{|l|}{ General health } \\
\hline Preoperative & $67.5 \pm 27.7$ & $76.2 \pm 17.4$ & 0.330 \\
\hline Last visit & $73.3 \pm 20.2$ & $72.6 \pm 20.3$ & 0.949 \\
\hline$p$-value & 0.364 & $0.015^{*}$ & \\
\hline \multicolumn{4}{|l|}{ Vitality } \\
\hline Preoperative & $66.4 \pm 24.5$ & $68.9 \pm 21.2$ & 0.735 \\
\hline Last visit & $72.8 \pm 20.9$ & $73.3 \pm 19.2$ & 0.883 \\
\hline$p$-value & $0.003^{*}$ & $0.003^{*}$ & \\
\hline \multicolumn{4}{|l|}{ Social function } \\
\hline Preoperative & $65.7 \pm 36.6$ & $59.4 \pm 38.6$ & 0.493 \\
\hline Last visit & $88.1 \pm 26.4$ & $96.9 \pm 13.8$ & 0.084 \\
\hline$p$-value & $<0.001^{*}$ & 0.176 & \\
\hline \multicolumn{4}{|c|}{ Role limitations (emotional) } \\
\hline Preoperative & $84.6 \pm 32.3$ & $96.9 \pm 17.7$ & $0.034^{*}$ \\
\hline Last visit & $96.6 \pm 14.9$ & $98.4 \pm 8.8$ & 0.654 \\
\hline$p$-value & 0.121 & 0.861 & \\
\hline \multicolumn{4}{|l|}{ Mental health } \\
\hline Preoperative & $76.7 \pm 18.1$ & $79.3 \pm 19.8$ & 0.402 \\
\hline Last visit & $84.7 \pm 12.3$ & $85.9 \pm 13.1$ & 0.443 \\
\hline$p$-value & $0.044^{*}$ & $<0.001^{*}$ & \\
\hline
\end{tabular}

*Difference is statistically significant $(p<0.05)$. AOFAS: American Orthopaedic Foot and Ankle Society; CA: central approach; LA: lateral approach; SD: standard deviation; SF-36: 36-item Short Form Health Survey; VAS: visual analogue scale

The CA for IAT was first introduced by McGarvey et al in $2002^{(15)}$ and has gained more popularity in the past few years. It provides excellent visualisation of pathologies over the posterior heel and reduces the incidence of sural nerve injury. ${ }^{(20,21)}$ This
Table III. Postoperative complications and satisfaction rate.

\begin{tabular}{|llll|}
\hline & \multicolumn{2}{c}{ No. (\%) } & p-value \\
\cline { 2 - 3 } & $\begin{array}{l}\text { LA group } \\
\text { (n=39) }\end{array}$ & $\begin{array}{l}\text { CA group } \\
\text { (n= 32) }\end{array}$ & \\
\hline Postoperative complication & & & 0.813 \\
\hline Painful scar & $3(7.7)$ & $2(6.3)$ & 0.406 \\
\hline $\begin{array}{l}\text { Wound sensation } \\
\text { impairment }\end{array}$ & $3(7.7)$ & $1(3.1)$ & 0.049 \\
\hline Delayed wound healing & $1(2.6)$ & $5(15.6)$ & 0.362 \\
\hline $\begin{array}{l}\text { Superficial wound infection } \\
\text { Overgrown osteophyte }\end{array}$ & $1(2.6)$ & $0(0)$ & 0.362 \\
\hline Overall complication rate & $9(2.6)$ & $0(0)$ & 0.850 \\
\hline Satisfaction rate & $34(87.2)$ & $28(87.5)$ & 0.968 \\
\hline
\end{tabular}

CA: central approach; LA: lateral approach

approach also allows surgeons to treat intratendinous lesions or simultaneously perform a tendon release. ${ }^{(22,23)}$ A cadaveric study ${ }^{(24)}$ showed that the central incision lies between the peroneal angiosome and the posterior tibial angiosome; this may minimise vascular compromise from the heel surgery. However, one of the drawbacks of the CA is that it results in substantial tension over the wound after closure and, therefore, may cause delayed wound healing and wound dehiscence, as were commonly reported in previous studies. ${ }^{(15,20,21,25,26)}$ Similarly, we observed in our study that the CA led to significantly more cases of delayed wound healing when compared with the LA.

Both the LA and CA have been demonstrated to be effective. In the LA group, there was significant improvement in pain (i.e. mean postoperative scores), AOFAS Ankle-Hindfoot Scale scores and SF-36 scores in our study, and the findings were similar to those reported in previous studies. ${ }^{(9,14,19,27)}$ Similarly, significant improvements in pain and function were noted in the CA group, and the results were in agreement with observations reported by previous studies. ${ }^{(20,25,28,29)}$ The two approaches were comparable in effectiveness in improving pain scores, AOFAS Ankle-Hindfoot Scales and SF-36 scores, and this is consistent with the findings of Anderson et al. ${ }^{(30)}$ In our study, the satisfaction rate of surgery using the CA was $87.5 \%$, which falls within the range of $77 \%-100 \%$ reported in the literature. ${ }^{(15,28,29,31)}$ The satisfaction rate with the LA was $87.2 \%$ with no significant difference between the two approaches in terms of subjective evaluation.

Interestingly, we observed that the CA resulted in significantly more short-term pain when compared with the LA. However, both approaches were comparable in long-term pain relief. Although this may not be clinically significant, one possible explanation is that short-term heel pain may be related to the position and condition of the surgical wound, as recovery from open retrocalcaneal decompression is usually prolonged. ${ }^{(20,27)}$ The surgical wound from the CA lies posteriorly against the heel counter of footwear and tends to be more irritated by the pressure, particularly when the wound is still oedematous during recovery. However, once the patient has completely recovered, the wound from the CA does not cause more pain when compared to that from the LA.

The results of the current study need to be interpreted in light of its limitations. First, the follow-up duration is relatively short. 
Previous studies suggested that patients who underwent surgical correction for Haglund's deformity took six months to two years to recover. ${ }^{(27,32)}$ A longer follow-up duration may be required to evaluate the long-term outcomes of different approaches. Second, the number of patients in this study is relatively small, and the sample size may be not large enough to detect any potential difference between the two surgical approaches. Last, this is a retrospective study from a single institution, which may result in selection and observational biases. A prospective multicentre study with a longer follow-up duration and larger sample size is required to further evaluate the advantages and disadvantages of the LA or CA for operative management of IAT.

In conclusion, the LA and the CA with the operative techniques we described were effective treatments for IAT that resulted in similar functional improvement, long-term pain relief and overall enhancement of patients' health conditions. The two approaches were comparable in overall postoperative complication rate. With meticulous soft tissue handling, the LA provided better pain relief in the short term and reduced delayed wound healing as compared with the CA.

\section{REFERENCES}

1. Maffulli N, Khan KM, Puddu G. Overuse tendon conditions: time to change a confusing terminology. Arthroscopy 1998; 14:840-3.

2. Rio E, Mayes S, Cook J. Heel pain: a practical approach. Aust Fam Physician 2015; 44:96-101.

3. Lysholm J, Wiklander J. Injuries in runners. Am J Sports Med 1987; 15:168-71.

4. Irwin T. Current concepts review: insertional achilles tendinopathy. Foot Ankle Int 2010; 31:933-9.

5. Bah I, Kwak ST, Chimenti RL, et al. Mechanical changes in the Achilles tendon due to insertional Achilles tendinopathy. J Mech Behav Biomed Mater 2016; 53:320-8.

6. Li HY, Hua YH. Achilles tendinopathy: current concepts about the basic science and clinical treatments. Biomed Res Int 2016; 2016:6492597.

7. Maffulli N, Sharma P, Luscombe KL. Achilles tendinopathy: aetiology and management. J R Soc Med 2004; 97:472-6.

8. Sundararajan PP, Wilde TS. Radiographic, clinical, and magnetic resonance imaging analysis of insertional Achilles tendinopathy. J Foot Ankle Surg 2014; 53:147-51.

9. Oshri Y, Palmanovich E, Brin YS, et al. Chronic insertional Achilles tendinopathy: surgical outcomes. Muscles Ligaments Tendons J 2012; 2:91-5.

10. Scott A, Huisman E, Khan K. Conservative treatment of chronic Achilles tendinopathy. CMAJ 2011; 183:1159-65.

11. Tu P, Bytomski J. Diagnosis of heel pain. Am Fam Physician 2011; 84:909-16.

12. Wiegerinck JI, Kerkhoffs GM, van Sterkenburg MN, Sierevelt IN, van Dijk CN. Treatment for insertional Achilles tendinopathy: a systematic review. Knee Surg Sports Traumatol Arthrosc 2013; 21:1345-55.
13. Witt BL, Hyer CF. Achilles tendon reattachment after surgical treatment of insertional tendinosis using the suture bridge technique: a case series. J Foot Ankle Surg 2012; 51:487-93.

14. Lin HA, Chong HA, Yeo W. Calcaneoplasty and reattachment of the Achilles tendon for insertional tendinopathy. J Orthop Surg (Hong Kong) 2014; 22:56-9.

15. McGarvey WC, Palumbo RC, Baxter DE, Leibman BD. Insertional Achilles tendinosis: surgical treatment through a central tendon splitting approach. Foot Ankle Int 2002; 23:19-25.

16. Xia Z, Yew AKS, Zhang TK, et al. Surgical correction of Haglund's triad using a central tendon-splitting approach: a retrospective outcomes study. J Foot Ankle Surg 2017; 56:1132-8.

17. Palmer I. The mechanism and treatment of fractures of the calcaneus; open reduction with the use of cancellous grafts. J Bone Joint Surg Am 1948; 30A:2-8.

18. Freeman BJ, Duff S, Allen PE, Nicholson HD, Atkins RM. The extended lateral approach to the hindfoot. Anatomical basis and surgical implications. J Bone Joint Surg Br 1998; 80:139-42.

19. Yodlowski ML, Scheller AD Jr, Minos L. Surgical treatment of Achilles tendinitis by decompression of the retrocalcaneal bursa and the superior calcaneal tuberosity. Am J Sports Med 2002; 30:318-21.

20. Johnson KW, Zalavras C, Thordarson DB. Surgical management of insertional calcific achilles tendinosis with a central tendon splitting approach. Foot Ankle Int 2006; 27:245-50.

21. Highlander P, Greenhagen RM. Wound complications with posterior midline and posterior medial leg incisions: a systematic review. Foot Ankle Spec 2011; 4:361-9.

22. Miao XD, Jiang $H, W u$ YP, et al. Treatment of calcified insertional Achilles tendinopathy by the posterior midline approach. J Foot Ankle Surg 2016; 55:529-34

23. Oliva F, Via AG, Maffulli N. Physiopathology of intratendinous calcific deposition. BMC Med 2012; 10:95.

24. Attinger CE, Evans KK, Bulan E, Blume P, Cooper P. Angiosomes of the foot and ankle and clinical implications for limb salvage: reconstruction, incisions, and revascularization. Plast Reconstr Surg 2006; 117(7 Suppl):261S-93S.

25. Gillis CT, Lin JS. Use of a central splitting approach and near complete detachment for insertional calcific Achilles tendinopathy repaired with an Achilles bridging suture. J Foot Ankle Surg 2016; 55:235-9.

26. McAlister JE, Hyer CF. Safety of Achilles detachment and reattachment using a standard midline approach to insertional enthesophytes. J Foot Ankle Surg 2015; 54:214-9.

27. Brunner J, Anderson J, O'Malley M, et al. Physician and patient based outcomes following surgical resection of Haglund's deformity. Acta Orthop Belg 2005; 71:718-23.

28. Ettinger S, Razzaq R, Waizy H, et al. Operative treatment of the insertional Achilles tendinopathy through a transtendinous approach. Foot Ankle Int 2016; 37:288-93.

29. Ahn JH, Ahn CY, Byun CH, Kim YC. Operative treatment of Haglund syndrome with central Achilles tendon-splitting approach. J Foot Ankle Surg 2015; 54:1053-6.

30. Anderson JA, Suero E, O'Loughlin PF, Kennedy JG. Surgery for retrocalcaneal bursitis: a tendon-splitting versus a lateral approach. Clin Orthop Relat Res 2008; 466:1678-82

31. Nunley JA, Ruskin G, Horst F. Long-term clinical outcomes following the central incision technique for insertional Achilles tendinopathy. Foot Ankle Int 2011; 32:850-5.

32. Schneider W, Niehus W, Knahr K. Haglund's syndrome: disappointing results following surgery - a clinical and radiographic analysis. Foot Ankle Int 2000; 21:26-30. 